\title{
TRIPLE CANONICAL COVERS OF VARIETIES OF MINIMAL DEGREE
}

\author{
Francisco Javier Gallego \\ AND \\ Bangere P. Purnaprajna
}

\begin{abstract}
In this article we study pluriregular varieties $X$ of general type with base-point-free canonical bundle whose canonical morphism has degree 3 and maps $X$ onto a variety of minimal degree $Y$. We carry out our study from two different perspectives.

First we study in Section 2 the canonical ring of $X$ describing completely the degrees of its minimal generators. We apply this to the study of the projective normality of the images of the pluricanonical morphisms of $X$. Our study of the canonical ring of $X$ also shows that, if the dimension of $X$ is greater than or equal to 3 , there does not exist a converse to a theorem of M. Green that bounds the degree of the generators of the canonical ring of $X$. This is in sharp contrast with the situation in dimension 2 where such converse exists, as proved by the authors in a previous work.

Second, we study in Section 3, the structure of the canonical morphism of $X$. We use this to show among other things the non-existence of some a priori plausible examples of triple canonical covers of varieties of minimal degree. We also characterize the targets of flat canonical covers of varieties of minimal degree. Some of the results of Section 3 are more general and apply to varieties $X$ which are not necessarily regular, and to targets $Y$ that are scrolls which are not of minimal degree.
\end{abstract}

MSC: 14J30, 14J35, 14J40, 14J29, 14E20

The first author was partially supported by MCT project number BFM2000-0621 and by UCM project number PR52/00-8862. The second author was partially supported by the General Research Fund of the University of Kansas at Lawrence. The first author is grateful for the hospitality of the Department of Mathematics of the University of Kansas at Lawrence.

Typeset by $\mathcal{A M}_{\mathcal{M}} \mathrm{T}_{\mathrm{E}} \mathrm{X}$ 


\section{INTRODUCTION}

The purpose of the article is to study pluriregular varieties (these are, according to Catanese, varieties such that the intermediate cohomology of its structure sheaf vanish) of general type $X$ with base-point-free canonical divisor and such that the canonical morphism $\pi$ is a generically $3: 1$ morphism onto a variety of minimal degree $Y$. The varieties of general type whose canonical morphism maps to a variety of minimal degree play an important role in various settings. In dimension 2 they are central in the classification of surfaces of general type with small $c_{1}^{2}$ and in questions about degenerations and the moduli of surfaces of general type. This is illustrated by the works of Horikawa, Catanese, Konno, Mendes Lopes and Pardini, among others. In higher dimensions, pluriregular varieties of general type have been studied by F. Catanese (see [Ca1] and [Ca2]) and M. Green $([\mathrm{G}])$ who prove results on the canonical ring of these varieties.

We study these varieties from two different perspectives. The first perspective we take is the study of the structure of their canonical ring. This is handled in Section 2. More precisely our main goal is to find out the degrees of the generators. This goal is accomplished in Theorem 2.6 for odd dimensional varieties and Theorem 2.11 for even dimensional varieties, where we obtain a full description of the degrees in terms of the dimension of $X$ and the degree of $Y$. We put these two results together in the following

Theorem 1. Let $X$ be a smooth, pluriregular variety of general type of dimension $m \geq 2$ and let $Y \subset \mathbf{P}^{r+m-1}$ be an $m$-fold of minimal degree. Assume that $K_{X}$ is base-point-free and let $\pi: X \longrightarrow Y$ be the canonical morphism of $X$. Assume that $\pi$ is a generically finite morphism of degree 3 . Then

1) if $m$ is odd, the canonical ring of $X$ is generated by its part of degree 1 and $\frac{r}{2}$ linearly indendent generators in degree $\frac{m+1}{2}$;

2) if $m$ is even, the canonical ring of $X$ is generated by its elements of degree $1, r$ linearly independent generators in degree $\frac{m+2}{2}$ and $r-1$ linearly independent generators in degree $m+1$.

Apart from its intrinsic interest, Theorem 1 has several applications. In the first place the degrees of the generators of the canonical ring are related to the degrees of the generators of the pluricanonical rings and therefore, with the very ampleness and projective normality associated to pluricanonical morphisms on the variety $X$. In this regard, using Theorem 1 (1) for the odd dimensional case, we give a necessary and sufficient condition for multiples of the canonical bundle 
$K_{X}$ on $X$ to give projectively normal embeddings. Precisely (see Theorem 2.9) we obtain

Let $X$ an odd dimensional variety satisfying the hypothesis of Theorem 1 . Assume in addition that $K_{X}$ is ample. Then the line bundle $K_{X}^{\otimes n}$ is very ample and the image of the morphism it induces is projectively normal if and only if $n \geq \frac{m+1}{2}$.

In the even dimensional case using Theorem 1 (2) we obtain a sufficient condition for a multiple of $K_{X}$ to induce a projectively normal embedding (see Theorem 2.13).

Another interesting consequence of Theorem 1 concerns this general question: "what is the minimal degree of the generators of the canonical ring of a variety of general type?." Green (see [G]) proved that the canonical ring of a pluriregular variety of general type is generated in degree less than or equal to $n$ if the canonical morphism does not map $X$ onto a variety of minimal degree. Here is yet another instance where varieties of minimal degree play an important role. For surfaces, the above mentioned result by Green was also obtained independently by Ciliberto (see $[\mathrm{Ci}]$ ). In [GP] we proved a converse of this result of Green and Ciliberto. Precisely, we found out the degrees of the generators of the canonical ring of a regular surface of general type $X$ whose canonical morphism mapped $X$ to a surface of minimal degree. As a corollary of this we proved (cf. [GP], Corollary 2.8) a converse to the result of Ciliberto and Green. For higher dimensions, Theorem 1 and the examples at the end of Section 3 draw a very different picture. Indeed Theorem 1 and Proposition 3.4 show that a converse to Green's result, that is, a result saying that the canonical ring of a pluriregular variety of general type is generated in degree less than or equal to $n$ if and only if the canonical morphism does not map $X$ onto a variety of minimal degree, is false in dimension greater than 2. The reason the converse fails is due to the fact that for the varieties studied in Section 2, the canonical ring is generated in degree much lower than the Green's bound, roughly, in degree half of Green's bound. This fact is noted in Remark 3.5.

The second approach we take is to study the structure of the canonical morphism of varieties $X$ of general type which are triple covers of varieties of minimal degree $Y$. This is dealt with in Section 3. We will mention here some of the interesting consequences of this study. We obtain results which show that the fact 
of working with a morphism induced by a canonical subseries imposes strong restrictions on the parity of the dimension of $X$ and $Y$ and of the degree of $Y$. These results hold for varieties of general type which are more general than those considered in Section 1. For instance, as a consequence of Theorem 3.2, we obtain this result which holds for canonical morphisms of arbitrary degree and for any scroll $Y$ not necessarily fibered over $\mathbf{P}^{1}$ :

(1) An even dimensional variety of general type does not admit a generically finite canonical morphism of odd degree to a scroll.

When the canonical morphism is generically finite of degree 3, we go further in our study and obtain Theorem 3.1 and Theorem 3.3. We mention below some of the consequences of these theorems:

(2) We show that there is a relation between the dimension $m$ of $X$ and the degree $r$ of $Y$. More precisely, we see that the condition of $m$ being odd forces $r$ to be even. In particular there are no odd dimensional varieties of general type which are triple canonical covers of linear $\mathbf{P}^{m}$.

With the additional hypothesis of flatness on the canonical morphism, we obtain the following stronger results:

(3) A variety of general type which is a canonical triple cover of a variety of minimal degree is pluriregular (see Theorem 3.1).

(4) Theorem 3.3 shows that all triple flat canonical covers $X \stackrel{\pi}{\rightarrow} Y$ of varieties of minimal degree are such that $\pi_{*} \mathcal{O}_{X}$ splits as vector bundle over $\mathcal{O}_{Y}$ in the same way as a cyclic cover.

(5) We determine precisely what are the varieties of general type which occur as targets of canonical morphisms of degree 3. This is achieved by Theorem 3.3 and Proposition 3.4.

Moreover, the pluriregular varieties of general type constructed in Proposition 3.4 illustrate the theorems that we prove in this paper.

We will say now a few words on the techniques used to prove the results of Section 2. These techniques involve the study of the $\mathcal{O}_{Y}$-algebra structure on $\pi_{*} \mathcal{O}_{X}$ to find the multiplicative structure of the canonical ring of $X$. Even though we reduce the problem from a complicated variety to a simpler variety, as a variety of minimal degree $Y$ appear to be when compared to the variety $X$, there are natural difficulties that arise in the process. The proof of Theorem 
2.6 and Theorem 2.11 involves the study of multiplication of global sections of line bundles on the $m$-dimensional variety of general type $X$. To do so we relate the problem of multiplication of global sections of line bundles on $X$ to that of multiplication of global sections of line bundles on a curve $C$ which is an $m-1$ complete intersection on $X$. We achieve this through an inductive process that we develop in Lemma 2.3. An impression that things become simple on curves is misleading since by a process such as this one obtains bundles of small degree on $C$ which are difficult to handle in the context of multiplication maps. To circumvent this we transfer the problem of multiplication maps on $C$ to a rational normal curve obtained as $m-1$ complete intersection on $Y$ by means of pushing down from $C$ by $\pi$. We have traded one difficulty, that of handling small degree bundles on the curve $C$ of $X$, to another, namely, the study of certain maps on $\mathbf{P}^{1}$. These maps involve global sections of vector bundles on $\mathbf{P}^{1}$ but are not multiplication maps of global sections. The key ingredient to this approach, which allows us to settle the problem, is to use the algebra structure of $\mathcal{O}_{C}$ over $\mathcal{O}_{\mathbf{P}^{1}}$ to interpret the maps appearing on $\mathbf{P}^{1}$ and to see their relation with the multiplication maps on $C$. Note finally that, unlike double covers, a triple cover is not determined by the branch locus and the algebra structure it induces on $\mathcal{O}_{X}$ might be quite complicated. This is another difficulty one encounters but we are able to overcome it in the context of this paper.

Convention. Throughout this article the base field $\mathbf{k}$ has characteristic 0 and $\overline{\mathbf{k}}=\mathbf{k}$. By variety we will mean an irreducible projective variety over $\mathbf{k}$.

\section{The CANONicAl Ring of tRIPLE COVERS OF VARIETIES OF MINIMAL DEGREE}

The purpose of this section is to study the canonical ring of a pluriregular variety $X$ of general type with base-point-free canonical bundle $K_{X}$ and such that its canonical morphism maps $X$ generically $3: 1$ onto a variety of minimal degree. Precisely we want to prove Theorem 1 of the introduction, which tells the degrees of the generators of the canonical ring of $X$. To do so we will need to establish some notation and to prove some auxiliary lemmas and results.

Notation 2.1. Let $X$ be a smooth variety of general type of dimension $m$ and let $K_{X}$ be its canonical bundle. Assume that $K_{X}$ is base-point-free. Let $X_{1} \subset \cdots \subset X_{m^{\prime}} \subset \cdots X_{m}=X$ be smooth irreducible subsequent $m^{\prime}$ dimensional, complete intersections of members of $\left|K_{X}\right|$. We will also denote 
$X_{1}$ by $C$. We denote $\left.K_{X}\right|_{X_{m^{\prime}}}$ by $L_{m^{\prime}}$ (in particular $L_{m}=K_{X}$ ) and we will also denote $L_{1}$ by $\theta$. Finally let

$$
H^{0}\left(X_{m^{\prime}}, L_{m^{\prime}}^{\otimes s}\right) \otimes H^{0}\left(X_{m^{\prime}}, L_{m^{\prime}}^{\otimes t}\right) \stackrel{\alpha\left(s, t ; m^{\prime}\right)}{\longrightarrow} H^{0}\left(X_{m^{\prime}}, L_{m^{\prime}}^{\otimes s+t}\right)
$$

be the usual multiplication map of global sections.

We will use also some abridged notation:

$$
\begin{aligned}
& \alpha(s, t ; m)=\alpha(s, t), \\
& \alpha(s, t ; 1)=\beta(s, t), \\
& \alpha\left(s, 1 ; m^{\prime}\right)=\alpha\left(s ; m^{\prime}\right) \\
& \alpha(s, 1 ; m)=\alpha_{s} \text { and } \\
& \alpha(s, 1 ; 1)=\beta_{s} .
\end{aligned}
$$

The idea of the proofs of Theorem 1 is to relate the computation of generators of the canonical ring to the knowledge of the images of certain maps of multiplication of global sections of line bundles on $X$. We will find out the image of these maps by studying analogous maps of multiplication of global sections of line bundles on the varieties $X_{m^{\prime}}$ defined above, and eventually, by studying maps on the curve $C$. In order to realize the link between the maps on $X_{m^{\prime}}$ and the maps on $X_{m}$ we need a couple of lemmas.

Lemma 2.2. Let $X$ be a smooth, pluriregular variety of general type of dimension $m$, such that its canonical bundle $K_{X}$ is base-point-free. Then:

1) $H^{b}\left(L_{m^{\prime}}^{\otimes a}\right)=0$ for all $2 \leq m^{\prime} \leq m, 1 \leq b \leq m^{\prime}-1$

2) The natural maps of restriction of global sections

$$
H^{0}\left(L_{m^{\prime}}^{\otimes n}\right) \longrightarrow H^{0}\left(L_{m^{\prime}-i}^{\otimes n}\right)
$$

surject for all $2 \leq m^{\prime} \leq m$, all $1 \leq i \leq m^{\prime}-1$ and all $n \geq 1$. In particular, the maps

$$
\begin{gathered}
H^{0}\left(L_{m^{\prime}}^{\otimes n}\right) \longrightarrow H^{0}\left(L_{m^{\prime}-1}^{\otimes n}\right) \text { and } \\
H^{0}\left(K_{X}^{\otimes n}\right) \longrightarrow H^{0}\left(\theta^{\otimes n}\right)
\end{gathered}
$$

surject for all $n \geq 1$.

Proof. If $\left.m^{\prime}=m, 1\right)$ follows from hypothesis, Kawamata-Viehweg vanishing and Serre duality. Arguing by induction on the codimension and using the long exact sequence of cohomology which arises from

$$
0 \longrightarrow L_{m^{\prime}}^{\otimes n-1} \longrightarrow L_{m^{\prime}}^{\otimes n} \longrightarrow L_{m^{\prime}-1}^{\otimes n} \longrightarrow 0
$$


we prove 1) for all $2 \leq m^{\prime} \leq m$. To prove 2 ) it is enough to show the surjectivity of

$$
H^{0}\left(L_{m^{\prime}}^{\otimes n}\right) \stackrel{\mu}{\rightarrow} H^{0}\left(L_{m^{\prime}-1}^{\otimes n}\right)
$$

for all $2 \leq m^{\prime} \leq m$ and $n \geq 1$, since the maps

$$
H^{0}\left(L_{m^{\prime}}^{\otimes n}\right) \longrightarrow H^{0}\left(L_{m^{\prime}-i}^{\otimes n}\right)
$$

are composite of maps like the previous one. But the surjectivity of $\mu$ follows at once from the vanishing of $H^{1}\left(L_{m^{\prime}}^{\otimes n-1}\right)$, which follows from 1).

Lemma 2.3. Let $X$ be a smooth, pluriregular variety of general type of dimension $m$, such that its canonical bundle $K_{X}$ is base-point-free.

1) Let $s_{1}, s_{2} \geq 1$. If

$$
\begin{gathered}
H^{0}\left(K_{X}^{\otimes s_{1}}\right) \otimes H^{0}\left(K_{X}^{\otimes s_{2}}\right) \stackrel{\alpha\left(s_{1}, s_{2} ; m\right)}{\longrightarrow} H^{0}\left(K_{X}^{\otimes s_{1}+s_{2}}\right) \text { surjects, so does } \\
H^{0}\left(\theta^{\otimes s_{1}}\right) \otimes H^{0}\left(\theta^{\otimes s_{2}}\right) \stackrel{\alpha\left(s_{1}, s_{2} ; 1\right)}{\longrightarrow} H^{0}\left(\theta^{\otimes s_{1}+s_{2}}\right) .
\end{gathered}
$$

2) Given $s_{1}, s_{2} \geq 1$, assume that the maps

$$
H^{0}\left(\theta^{\otimes s^{\prime}}\right) \otimes H^{0}\left(\theta^{\otimes s_{2}}\right) \stackrel{\alpha\left(s^{\prime}, s_{2} ; 1\right)}{\longrightarrow} H^{0}\left(\theta^{\otimes s^{\prime}+s_{2}}\right)
$$

surject for all $1 \leq s^{\prime} \leq s_{1}$. Then the maps

$$
H^{0}\left(L_{m^{\prime}}^{\otimes s^{\prime}}\right) \otimes H^{0}\left(L_{m^{\prime}}^{\otimes s_{2}}\right) \stackrel{\alpha\left(s^{\prime}, s_{2} ; m^{\prime}\right)}{\longrightarrow} H^{0}\left(L_{m^{\prime}}^{\otimes s^{\prime}+s_{2}}\right)
$$

surject for all $1 \leq s^{\prime} \leq s_{1}$ and all $1 \leq m^{\prime} \leq m$.

3) Given $s_{1}, s_{2} \geq 1$, assume that the maps

$$
H^{0}\left(\theta^{\otimes s^{\prime}}\right) \otimes H^{0}\left(\theta^{\otimes s_{2}}\right) \stackrel{\alpha\left(s^{\prime}, s_{2} ; 1\right)}{\longrightarrow} H^{0}\left(\theta^{\otimes s^{\prime}+s_{2}}\right)
$$

surject for all $1 \leq s^{\prime} \leq s_{1}-1$. Then the image of the map

$$
H^{0}\left(L_{m^{\prime}}^{\otimes s_{1}}\right) \otimes H^{0}\left(L_{m^{\prime}}^{\otimes s_{2}}\right) \stackrel{\alpha\left(s_{1}, s_{2} ; m^{\prime}\right)}{\longrightarrow} H^{0}\left(L_{m^{\prime}}^{\otimes s_{1}+s_{2}}\right)
$$

is the inverse image of the image of map

$$
H^{0}\left(\theta^{\otimes s_{1}}\right) \otimes H^{0}\left(\theta^{\otimes s_{2}}\right) \stackrel{\alpha\left(s_{1}, s_{2} ; 1\right)}{\longrightarrow} H^{0}\left(\theta^{\otimes s_{1}+s_{2}}\right)
$$


by the obvious restriction map

$$
H^{0}\left(L_{m^{\prime}}^{\otimes s_{1}+s_{2}}\right) \longrightarrow H^{0}\left(\theta^{\otimes s_{1}+s_{2}}\right)
$$

Moreover, the codimension of $\alpha\left(s_{1}, s_{2} ; m^{\prime}\right)$ in $H^{0}\left(L_{m^{\prime}}^{\otimes s_{1}+s_{2}}\right)$ is the same for all $1 \leq m^{\prime} \leq m$.

Proof. First we prove 1). Consider the following commutative diagram:

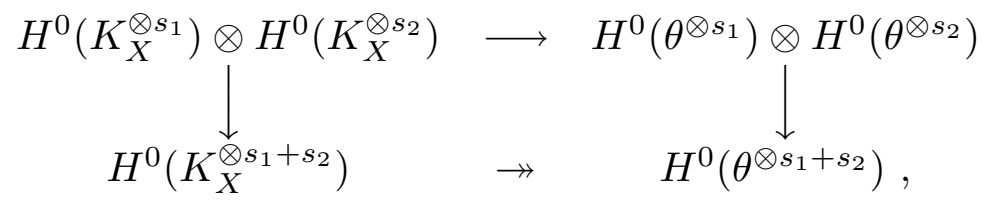

where the horizontal arrows are induced by restricting global sections from $X$ to $C$. The surjectivity of the bottom horizontal arrow follows from Lemma 2.2. Then it is clear that the surjectivity of the left hand side vertical arrow implies the surjectivity of right hand side vertical arrow.

Now we prove 2). Assume the maps

$$
H^{0}\left(\theta^{\otimes s^{\prime}}\right) \otimes H^{0}\left(\theta^{\otimes s_{2}}\right) \stackrel{\alpha\left(s^{\prime}, s_{2} ; 1\right)}{\longrightarrow} H^{0}\left(\theta^{\otimes s^{\prime}+s_{2}}\right)
$$

surject for all $1 \leq s^{\prime} \leq s_{1}$. We are going to show the surjectivity of $\alpha\left(s^{\prime}, s_{2} ; m^{\prime}\right)$ for all $1 \leq m^{\prime} \leq m$ and all $0 \leq s^{\prime} \leq s$. The proof is by induction on both on $m^{\prime}$. If $m^{\prime}=1$, the result is our hypothesis, the case $s^{\prime}=0$ being obvious. Now we assume the result true for $m^{\prime}-1$ and we will prove it for $m^{\prime}$ by induction on $s^{\prime}$. If $s^{\prime}=0$, the result is again obvious. Now we assume the result to be true for $s^{\prime}-1$ and we will prove it for $s^{\prime}$. Consider the following commutative diagram:

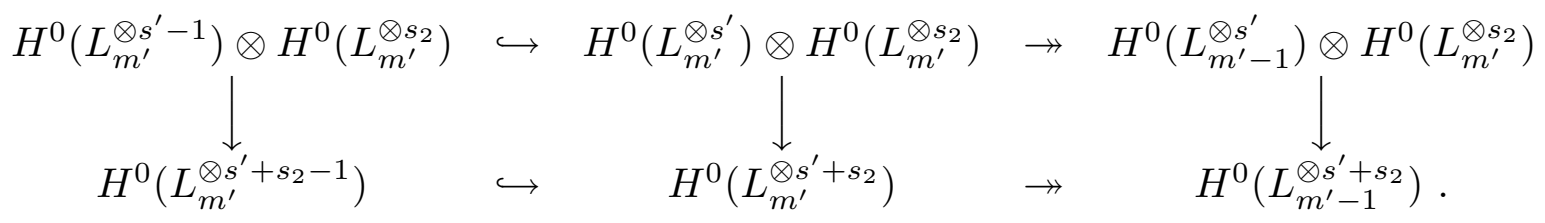

The last top and bottom horizonta 1 arrows are surjective by Lemma 2.2 . The left hand side vertical arrow is $\alpha\left(s^{\prime}-1, s_{2} ; m^{\prime}\right)$ and surjects by induction hypothesis on $s^{\prime}$. The right hand side vertical arrow is the composition of the map

$$
H^{0}\left(L_{m^{\prime}-1}^{\otimes s^{\prime}}\right) \otimes H^{0}\left(L_{m^{\prime}}^{\otimes s_{2}}\right) \longrightarrow H^{0}\left(L_{m^{\prime}-1}^{\otimes s^{\prime}}\right) \otimes H^{0}\left(L_{m^{\prime}-1}^{\otimes s_{2}}\right)
$$


whose surjectivity follows from Lemma 2.2, and the map of multiplication of global sections on $X_{m^{\prime}-1}, \alpha\left(s^{\prime}, s_{2} ; m^{\prime}-1\right)$, which is surjective by induction hypothesis on $m^{\prime}$. Then it follows from chasing the diagram that the middle vertical arrow $\alpha\left(s^{\prime}, s_{2} ; m^{\prime}\right)$ surjects.

Finally we prove 3 ) by induction on $m^{\prime}$. If $m^{\prime}=1$, there is nothing to prove. Now we assume the result true for $m^{\prime}-1$ with $m^{\prime} \geq 2$ and we will prove it for $m^{\prime}$. Consider the following commutative diagram:

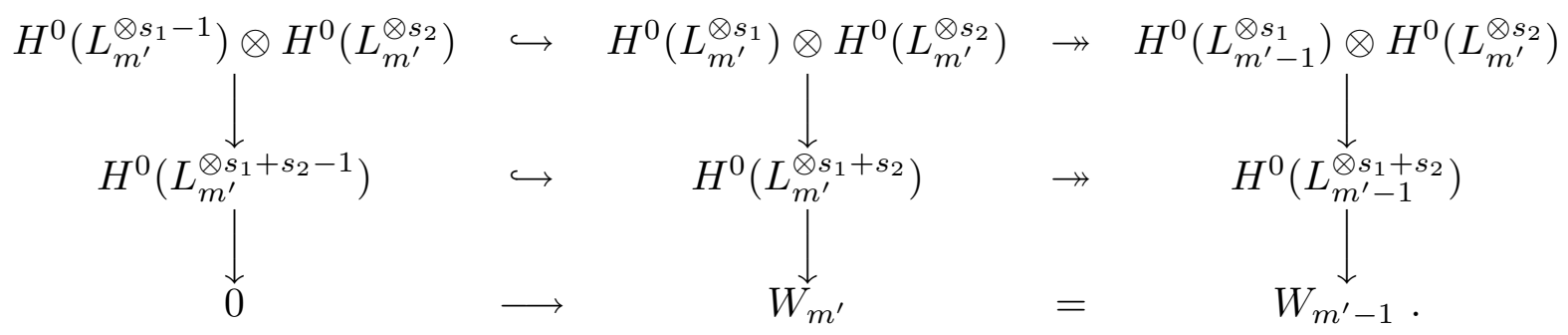

The last top and bottom horizontal arrows are surjective by Lemma 2.2. The left hand side vertical arrow is $\alpha\left(s_{1}-1, s_{2} ; m^{\prime}\right)$. This map surjects by part 2) of this lemma. The right hand side first vertical arrow is the composition of the map

$$
H^{0}\left(L_{m^{\prime}-1}^{\otimes s_{1}}\right) \otimes H^{0}\left(L_{m^{\prime}}^{\otimes s_{2}}\right) \longrightarrow H^{0}\left(L_{m^{\prime}-1}^{\otimes s_{1}}\right) \otimes H^{0}\left(L_{m^{\prime}-1}^{\otimes s_{2}}\right),
$$

and the map of multiplication of global sections on $X_{m^{\prime}-1}, \alpha\left(s_{1}, s_{2} ; m^{\prime}-1\right)$. The first of the two above mentioned maps is surjective by Lemma 2.2. Therefore the image of the right hand side first vertical arrow is equal to the image of $\alpha\left(s_{1}, s_{2} ; m^{\prime}-1\right)$. By induction hypothesis, the image of $\alpha\left(s_{1}, s_{2} ; m^{\prime}-1\right)$ is the inverse image of the image of $\alpha\left(s_{1}, s_{2} ; 1\right)$ by the natural map of restriction of global sections

$$
H^{0}\left(X_{m^{\prime}-1}, L_{m^{\prime}-1}^{\otimes s_{1}+s_{2}}\right) \longrightarrow H^{0}\left(C, \theta^{\otimes s_{1}+s_{2}}\right) .
$$

By chasing the diagram it follows that the image of $\alpha\left(s_{1}, s_{2}, m^{\prime}\right)$ is the inverse image of the image of $\alpha\left(s_{1}, s_{2}, m^{\prime}-1\right)$ by the restriction map

$$
H^{0}\left(X_{m^{\prime}}, L_{m^{\prime}}^{\otimes s_{1}+s_{2}}\right) \longrightarrow H^{0}\left(X_{m^{\prime}-1}, L_{m^{\prime}-1}^{\otimes s_{1}+s_{2}}\right) .
$$

So finally the image of $\alpha\left(s_{1}, s_{2} ; m^{\prime}\right)$ is the inverse image of the image of $\alpha\left(s_{1}, s_{2} ; 1\right)$ by the restriction map

$$
H^{0}\left(X_{m^{\prime}}, L_{m^{\prime}}^{\otimes s_{1}+s_{2}}\right) \longrightarrow H^{0}\left(C, \theta^{\otimes s_{1}+s_{2}}\right)
$$


Note finally that the isomorphism between $W_{m^{\prime}}$ and $W_{m^{\prime}-1}$ at each step of the inductive process tells us that the codimensions of the images of $\alpha\left(s_{1}, s_{2} ; m^{\prime}\right)$ in $H^{0}\left(X_{m^{\prime}}, L_{m^{\prime}}^{\otimes s_{1}+s_{2}}\right)$ are all equal for all $1 \leq m^{\prime} \leq m$.

As we have previously mentioned in order to prove Theorem 1 we need to study certain maps of multiplication of global sections of line bundles on the curve $C$ defined in Notation 2.1. We will prove Theorem 1 first when the dimension of $m$ is odd. On the curve $C$ we need the following

Proposition 2.4. Let $C$ be a smooth curve, let $\theta$ be an ample and base-pointfree line bundle on $C$ and let $m$ be an odd natural number such that $\theta^{\otimes m}=K_{C}$. Let $\varphi: X \longrightarrow Y$ be the morphism induced by a subseries $W^{\prime}$ of $|\theta|$ without base points, and assume that the degree of $\varphi$ is 3 and that $Y$ is a (smooth) rational normal curve of degree $r$. Then

1.1)

$$
\left.\varphi_{*}\left(\mathcal{O}_{C}\right)=\mathcal{O}_{\mathbf{P}^{1}} \oplus \mathcal{O}_{\mathbf{P}^{1}}\left(-\frac{m r+2}{2}\right) \oplus \mathcal{O}_{\mathbf{P}^{1}}(-m r-2)\right)
$$

In particular, $r$ is even.

1.2) If $m \geq 3$, then $\varphi$ is in fact induced by the complete linear series of $\theta$.

2.1) The map $\beta_{n}$ in $H^{0}\left(\theta^{\otimes n+1}\right)$ surjects if and only if $n \neq \frac{m-1}{2}, m$;

2.2) The map $\beta\left(\frac{m+1}{2}, l\right)$ surjects in $H^{0}\left(\theta^{\otimes \frac{m+1}{2}+l}\right)$ if $0 \leq l \leq \frac{m+1}{2}$.

2.3) The image of $\beta\left(s_{1}, s_{2}\right)$ is the same subspace $U^{\prime}$ of $H^{0}\left(\theta^{\otimes \frac{m+1}{2}}\right)$ for all

$s_{1}, s_{2}$ such that $s_{1}+s_{2}=\frac{m+1}{2}, s_{1}, s_{2} \geq 1$. The codimension of $U^{\prime} i s \frac{r}{2}$.

2.4) The map $\beta\left(s_{1}, s_{2}\right)$ surjects if $s_{1}, s_{2} \geq 0$ and $s_{1}+s_{2} \leq \frac{m-1}{2}$.

3) The ring $R_{\theta}=\bigoplus_{s=1}^{\infty}\left(R_{\theta}\right)_{s}$, where $\left(R_{\theta}\right)_{s}=H^{0}\left(\theta^{\otimes s}\right)$, is generated by its part of degree

1 and $\frac{r}{2}$ generators in degree $\frac{m+1}{2}$.

(2.5) Proof of Proposition 2.4. We first prove 1.1). Since $\varphi$ is finite and $Y$ is smooth, $\varphi$ is finite and flat, then $\varphi_{*}\left(\mathcal{O}_{C}\right)$ is a vector bundle of rank 3 . The bundle $\varphi_{*}\left(\mathcal{O}_{C}\right)$ splits as direct sum of line bundles over $\mathbf{P}^{1}$. Let

$$
\varphi_{*}\left(\mathcal{O}_{C}\right)=\mathcal{O}_{\mathbf{P}^{1}} \oplus \mathcal{O}_{\mathbf{P}^{1}}\left(-a_{1}\right) \oplus \mathcal{O}_{\mathbf{P}^{1}}\left(-a_{2}\right)
$$

for integers $a_{1} \leq a_{2}$, which are strictly positive because $C$ is connected, hence $h^{0}\left(\mathcal{O}_{C}\right)=1$. Recall that $K_{C}=\theta^{\otimes m}$ and that $\theta=\varphi^{*}\left(\mathcal{O}_{\mathbf{P}^{1}}(r)\right)$. Then, on the one hand,

$$
\varphi_{*}\left(K_{C}\right)=\mathcal{O}_{\mathbf{P}^{1}}(m r) \oplus \mathcal{O}_{\mathbf{P}^{1}}\left(m r-a_{1}\right) \oplus \mathcal{O}_{\mathbf{P}^{1}}\left(m r-a_{2}\right)
$$


by projection formula. On the other hand,

$$
\varphi_{*}\left(K_{C}\right)=\left(\varphi_{*}\left(\mathcal{O}_{C}\right)\right)^{*} \otimes K_{\mathbf{P}^{1}}=\mathcal{O}_{\mathbf{P}^{1}}\left(a_{2}-2\right) \oplus \mathcal{O}_{\mathbf{P}^{1}}\left(a_{1}-2\right) \oplus \mathcal{O}_{\mathbf{P}^{1}}(-2)
$$

by relative duality. Then

$$
\begin{gathered}
m r=a_{2}-2 \\
m r-a_{1}=a_{1}-2 \\
m r-a_{2}=-2,
\end{gathered}
$$

from where $a_{1}=\frac{m r+2}{2}$ and $a_{2}=m r+2$. Moreover, since $m$ is odd and $m r+2$ has to be even, $r$ is even. This completes the proof of 1.1).

Now we prove 1.2). It follows from 1.1) and projection formula that

$$
\begin{gathered}
H^{0}(\theta)=H^{0}\left(\varphi_{*}(\theta)\right)=H^{0}\left(\mathcal{O}_{\mathbf{P}^{1}}(r)\right) \\
\left.\left.\oplus \mathcal{O}_{\mathbf{P}^{1}}\left(\frac{(2-m) r-2}{2}\right) \oplus \mathcal{O}_{\mathbf{P}^{1}}((1-m) r-2)\right)\right) .
\end{gathered}
$$

Then $H^{0}\left(\varphi_{*}(\theta)\right)=H^{0}\left(\mathcal{O}_{\mathbf{P}^{1}}(r)\right)$, for $m \geq 3$. Therefore $\varphi$ is induced by the complete $|\theta|$.

We have seen that $\varphi_{*}\left(\mathcal{O}_{C}\right)$ and $\left.\mathcal{O}_{\mathbf{P}^{1}} \oplus \mathcal{O}_{\mathbf{P}^{1}}\left(-\frac{m r+2}{2}\right) \oplus \mathcal{O}_{\mathbf{P}^{1}}(-m r-2)\right)$ are isomorphic as sheaves of modules over $\mathcal{O}_{\mathbf{P}^{1}}$. We call $E=\mathcal{O}_{\mathbf{P}^{1}}\left(-\frac{m r+2}{2}\right) \oplus$ $\left.\mathcal{O}_{\mathbf{P}^{1}}(-m r-2)\right)$. Since $\varphi_{*}\left(\mathcal{O}_{C}\right)$ is also a sheaf of algebras over $\mathcal{O}_{\mathbf{P}^{1}}$, then the algebra structure of $\varphi_{*}\left(\mathcal{O}_{C}\right)$ makes $\left.\mathcal{O}_{\mathbf{P}^{1}} \oplus \mathcal{O}_{\mathbf{P}^{1}}\left(-\frac{m r+2}{2}\right) \oplus \mathcal{O}_{\mathbf{P}^{1}}(-m r-2)\right)$ into a

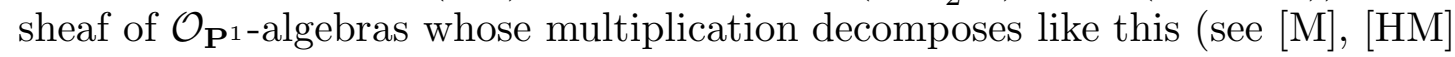
or $[\mathrm{GP}])$ : The map

$$
\mathcal{O}_{\mathbf{P}^{1}} \otimes \mathcal{O}_{\mathbf{P}^{1}} \longrightarrow \mathcal{O}_{\mathbf{P}^{1}}
$$

is the ring multiplication in $\mathcal{O}_{\mathbf{P}^{1}}$. The maps

$$
\begin{array}{clcr}
\mathcal{O}_{\mathbf{P}^{1}} \otimes \mathcal{O}_{\mathbf{P}^{1}}\left(-\frac{m r+2}{2}\right) & \longrightarrow & \mathcal{O}_{\mathbf{P}^{1}}\left(-\frac{m r+2}{2}\right) & \\
\mathcal{O}_{\mathbf{P}^{1}} \otimes \mathcal{O}_{\mathbf{P}^{1}}(-m r-2) & \longrightarrow & \mathcal{O}_{\mathbf{P}^{1}}(-m r+2) & \\
\mathcal{O}_{\mathbf{P}^{1}}\left(-\frac{m r+2}{2}\right) \otimes \mathcal{O}_{\mathbf{P}^{1}} & \longrightarrow & \mathcal{O}_{\mathbf{P}^{1}}\left(-\frac{m r+2}{2}\right) & \text { and } \\
\mathcal{O}_{\mathbf{P}^{1}}(-m r-2) \otimes \mathcal{O}_{\mathbf{P}^{1}} & \longrightarrow & \mathcal{O}_{\mathbf{P}^{1}}(-m r+2) &
\end{array}
$$

are the left and right module multiplication of $\mathcal{O}_{\mathbf{P}^{1}}\left(-\frac{m r+2}{2}\right)$ and $\mathcal{O}_{\mathbf{P}^{1}}(-m r+2)$. Finally there is a map

$$
E \otimes E \longrightarrow \varphi_{*} \mathcal{O}_{C}
$$


whose structure depends on the cover $\varphi$ itself. We will use all this to prove 2). Since $\theta=\varphi^{*} \mathcal{O}_{\mathbf{P}^{1}}(r)$, it follows from 1) and projection formula that

$H^{0}\left(\theta^{\otimes n}\right)=H^{0}\left(\mathcal{O}_{\mathbf{P}^{1}}(n r)\right) \oplus H^{0}\left(\mathcal{O}_{\mathbf{P}^{1}}\left(\frac{(2 n-m) r-2)}{2}\right)\right) \oplus H^{0}\left(\mathcal{O}_{\mathbf{P}^{1}}((n-m) r-2)\right)$.

Let us denote

$$
\begin{array}{ccc}
A(n) & = & H^{0}\left(\mathcal{O}_{\mathbf{P}^{1}}(n r)\right) \\
B(n) & = & H^{0}\left(\mathcal{O}_{\mathbf{P}^{1}}\left(\frac{(2 n-m) r-2)}{2}\right)\right) \\
C(n) & = & H^{0}\left(\mathcal{O}_{\mathbf{P}^{1}}((n-m) r-2)\right)
\end{array} .
$$

The way in which global sections of $H^{0}\left(\theta^{\otimes s_{1}}\right)$ and global sections of $H^{0}\left(\theta^{\otimes s_{2}}\right)$ multiply is governed by the ring multiplication in

$$
\left.\varphi_{*} \mathcal{O}_{C}=\mathcal{O}_{\mathbf{P}^{1}} \oplus \mathcal{O}_{\mathbf{P}^{1}}\left(-\frac{m r+2}{2}\right) \oplus \mathcal{O}_{\mathbf{P}^{1}}(-m r-2)\right)
$$

Thus the map $\beta\left(s_{1}, s_{2}\right)$ splits as direct sum of the following maps

$$
\begin{gathered}
A\left(s_{1}\right) \otimes A\left(s_{2}\right) \longrightarrow A\left(s_{1}+s_{2}\right) \\
A\left(s_{1}\right) \otimes B\left(s_{2}\right) \longrightarrow B\left(s_{1}+s_{2}\right) \\
A\left(s_{1}\right) \otimes C\left(s_{2}\right) \longrightarrow C\left(s_{1}+s_{2}\right) \\
B\left(s_{1}\right) \otimes A\left(s_{2}\right) \longrightarrow B\left(s_{1}+s_{2}\right) \\
C\left(s_{1}\right) \otimes A\left(s_{2}\right) \longrightarrow C\left(s_{1}+s_{2}\right) \\
{\left[B\left(s_{1}\right) \oplus C\left(s_{1}\right)\right] \otimes\left[B\left(s_{2}\right) \oplus C\left(s_{2}\right)\right] \longrightarrow} \\
A\left(s_{1}+s_{2}\right) \oplus B\left(s_{1}+s_{2}\right) \oplus C\left(s_{1}+s_{2}\right)(2.5 .1) .
\end{gathered}
$$

The first map $A\left(s_{1}\right) \otimes A\left(s_{2}\right) \longrightarrow A\left(s_{1}+s_{2}\right)$ is induced by the ring multiplication in $\mathcal{O}_{\mathbf{P}^{1}}$ and it is therefore a map of multiplication of global sections of line bundles on $\mathbf{P}^{1}$. The maps second to fifth are induced by the left and right module structure of $E$ over $\mathcal{O}_{\mathbf{P}^{1}}$ and are also multiplcation maps of global sections of line bundles on $\mathbf{P}^{1}$. The structure of the last map is more complex and depends on the structure of $\varphi$. Thus the image of $\beta\left(s_{1}, s_{2}\right)$ will be the sum of the images of those six maps. We assume $s_{1}, s_{2} \geq 0$. Then the first map is always nonzero and surjective, hence $A\left(s_{1}+s_{2}\right)$ is always contained in the image of $\beta\left(s_{1}, s_{2}\right)$. On the other hand the vanishing of the groups $B\left(s_{1}\right), B\left(s_{2}\right), B\left(s_{1}+s_{2}\right)$ and $C\left(s_{1}\right), C\left(s_{2}\right), C\left(s_{1}+s_{2}\right)$ depends on the values of $s_{1}$ and $s_{2}$. If $B\left(s_{1}\right)$ does not vanish, that is, if $s_{1} \geq \frac{m+1}{2}$, then $B\left(s_{1}+s_{2}\right)$ does not vanish either and the map

$$
B\left(s_{1}\right) \otimes A\left(s_{2}\right) \longrightarrow B\left(s_{1}+s_{2}\right)
$$


is surjective. Therefore in this case $B\left(s_{1}+s_{2}\right)$ is contained also in the image of $\beta\left(s_{1}, s_{2}\right)$. If $C\left(s_{1}\right)$ does not vanish, that is, if $s_{1} \geq m+1$, then $C\left(s_{1}+s_{2}\right)$ does not vanish either and the map

$$
C\left(s_{1}\right) \otimes A\left(s_{2}\right) \longrightarrow C\left(s_{1}+s_{2}\right)
$$

is surjective. Therefore in this case $C\left(s_{1}+s_{2}\right)$ is contained in the image of $\beta\left(s_{1}, s_{2}\right)$. There are analogous statements for $B\left(s_{2}\right)$ and $C\left(s_{2}\right)$.

With this information we prove now 2.1), 2.2), 2.3) and 2.4). We prove first 2.1). The multiplication map $\beta_{n}=\beta(n, 1)$ splits as shown in (2.5.1). If $0 \leq$ $n<\frac{m-1}{2}, B(n), C(n), B(n+1)$ and $C(n+1)$ all vanish, therefore $H^{0}\left(\theta^{\otimes n+1}\right)=$ $A(n+1)$, and the map

$$
H^{0}\left(\theta^{\otimes n}\right) \otimes H^{0}(\theta) \stackrel{\beta_{n}}{\longrightarrow} H^{0}\left(\theta^{\otimes n+1}\right)
$$

is the map

$$
A(n) \otimes A(1) \longrightarrow A(n+1)
$$

which is surjective. On the other hand, $B\left(\frac{m-1}{2}\right)=C\left(\frac{m-1}{2}\right)=C\left(\frac{m+1}{2}\right)=0$ but $B\left(\frac{m+1}{2}\right)$ has dimension $\frac{r}{2}>0$. Then $H^{0}(\theta)=A(1), H^{0}\left(\theta^{\otimes \frac{m-1}{2}}\right)=A\left(\frac{m-1}{2}\right)$ and $H^{0}\left(\theta^{\otimes \frac{m+1}{2}}\right)=A\left(\frac{m+1}{2}\right) \oplus B\left(\frac{m+1}{2}\right)$. Then

$$
H^{0}\left(\theta^{\otimes \frac{m-1}{2}}\right) \otimes H^{0}(\theta) \stackrel{\beta_{\frac{m-1}{2}}}{\longrightarrow} H^{0}\left(\theta^{\otimes \frac{m+1}{2}}\right)
$$

is the map

$$
A\left(\frac{m-1}{2}\right) \otimes A(1) \longrightarrow A\left(\frac{m+1}{2}\right)
$$

whose image has codimension $\frac{r}{2}$ in $H^{0}\left(\theta^{\otimes \frac{m+1}{2}}\right)$. If $\frac{m+1}{2} \leq n \leq m-1$, then $H^{0}\left(\theta^{\otimes n}\right)=A(n) \oplus B(n)$ and $H^{0}\left(\theta^{\otimes n+1}\right)=A(n+1) \oplus B(n+1)$, with $A(n), B(n)$, $A(n+1)$ and $B(n+1)$ all different from 0 . Then according to $((2.5 .1))$ if $\frac{m+1}{2} \leq n \leq m-1$ the map $\beta_{n}$ decomposes as direct sum of

$$
\begin{gathered}
A(n) \otimes A(1) \longrightarrow A(n+1) \text { and } \\
B(n) \otimes A(1) \longrightarrow B(n+1),
\end{gathered}
$$


which are surjective maps, therefore the image of $\beta_{n}$ is $A(n+1) \oplus B(n+1)=$ $H^{0}\left(\theta^{\otimes n+1}\right)$. On the other hand $C(m)=0$ but $C(m+1)$ has dimension $r-1>0$. Thus $H^{0}\left(\theta^{\otimes m}\right)=A(m) \oplus B(m)$ but

$$
H^{0}\left(\theta^{\otimes m+1}\right)=A(m+1) \oplus B(m+1) \oplus C(m+1) .
$$

The map $\beta_{m}$ decomposes as direct sum of

$$
\begin{gathered}
A(m) \otimes A(1) \longrightarrow A(m+1) \text { and } \\
B(m) \otimes A(1) \longrightarrow B(m+1),
\end{gathered}
$$

and then its image is $A(m+1) \oplus B(m+1)$, which has codimension $r-1$ in $H^{0}\left(\theta^{\otimes m+1}\right)$. Finally if $n \geq m+1, H^{0}\left(\theta^{n}\right)=A(n) \oplus B(n) \oplus C(n)$, and $A(n), B(n)$ and $C(n)$ are all nonzero. Then $\beta_{n}$ decomposes as direct sum of

$$
\begin{gathered}
A(n) \otimes A(1) \longrightarrow A(n+1) \\
B(n) \otimes A(1) \longrightarrow B(n+1) \\
C(n) \otimes A(1) \longrightarrow C(n+1),
\end{gathered}
$$

and its image is $A(n+1) \oplus B(n+1) \oplus C(n+1)=H^{0}\left(\theta^{n+1}\right)$. This completes the proof of 2.1).

Now we prove 2.2). Recall that if $\frac{m+1}{2} \leq n \leq m$, then $H^{0}\left(\theta^{\otimes n}\right)=A(n) \oplus B(n)$ and that, if $0 \leq l \leq \frac{m-1}{2}$, then $H^{0}\left(\theta^{\otimes l}\right)=A(l)$. As we argued in the proof of 2.1 , the map $\beta\left(\frac{m+1}{2}, l\right)$ is governed by the algebra structure of $\varphi_{*} \mathcal{O}_{C}$. Then, if $0 \leq l \leq \frac{m-1}{2}, \beta\left(\frac{m+1}{2}, l\right)$ decomposes as direct sum of

$$
\begin{gathered}
A\left(\frac{m+1}{2}\right) \otimes A(l) \longrightarrow A\left(\frac{m+1}{2}+l\right) \\
B\left(\frac{m+1}{2}\right) \otimes A(l) \longrightarrow B\left(\frac{m+1}{2}+l\right) .
\end{gathered}
$$

Then the image of $\beta\left(\frac{m+1}{2}, l\right)$ is $A\left(\frac{m+1}{2}+l\right) \oplus B\left(\frac{m+1}{2}+l\right)=H^{0}\left(\theta^{\otimes \frac{m+1}{2}+l}\right)$.

On the other hand, $H^{0}\left(\theta^{\otimes \frac{m+1}{2}}\right)=A\left(\frac{m+1}{2}\right) \oplus B\left(\frac{m+1}{2}\right)$. Therefore the map 
$\beta\left(\frac{m+1}{2}, \frac{m+1}{2}\right)$ decomposes as direct sum of

$$
\begin{gathered}
A\left(\frac{m+1}{2}\right) \otimes A\left(\frac{m+1}{2}\right) \longrightarrow A(m+1) \\
B\left(\frac{m+1}{2}\right) \otimes A\left(\frac{m+1}{2}\right) \longrightarrow B(m+1) \\
A\left(\frac{m+1}{2}\right) \otimes B\left(\frac{m+1}{2}\right) \longrightarrow B(m+1) \\
B\left(\frac{m+1}{2}\right) \otimes B\left(\frac{m+1}{2}\right) \stackrel{\eta}{\longrightarrow} \\
A(m+1) \oplus B(m+1) \oplus C(m+1) \quad(2.5 .2) .
\end{gathered}
$$

Since the first three maps are surjective We know that $A(m+1) \oplus B(m+1)$ is contained in the image of $\beta\left(\frac{m+1}{2}, \frac{m+1}{2}\right)$. Now we need to look more carefully at the last one of the above maps. For this we have to study more closely the ring structure of $\varphi_{*}\left(\mathcal{O}_{C}\right)$. Let

$$
\varphi_{*}\left(\mathcal{O}_{C}\right) \otimes \varphi_{*}\left(\mathcal{O}_{C}\right) \stackrel{\mu}{\rightarrow} \varphi_{*}\left(\mathcal{O}_{C}\right)
$$

be the map defined by the ring multiplication in $\varphi_{*}\left(\mathcal{O}_{C}\right)$. Then the projection to $\mathcal{O}_{\mathbf{P}^{1}}(-m r-2)$ of the restriction of $\mu$ to $\left.\mathcal{O}_{\mathbf{P}^{1}}\left(\frac{-m r-2}{2}\right) \otimes \mathcal{O}_{\mathbf{P}^{1}}\left(\frac{-m r-2}{2}\right)\right)$ is nonzero.

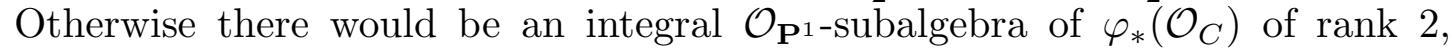
namely $\mathcal{O}_{\mathbf{P}^{1}} \oplus \mathcal{O}_{\mathbf{P}^{1}}\left(\frac{-m r-2}{2}\right)$. This is impossible because the rank of an integral subalgebra of $\varphi_{*} \mathcal{O}_{C}$ must divide the rank of $\varphi_{*} \mathcal{O}_{C}$. Now if the projection to $\mathcal{O}_{\mathbf{P}^{1}}(-m r-2)$ of the restriction of $\mu$ to $\left.\mathcal{O}_{\mathbf{P}^{1}}\left(\frac{-m r-2}{2}\right) \otimes \mathcal{O}_{\mathbf{P}^{1}}\left(\frac{-m r-2}{2}\right)\right)$ is nonzero, it is an isomorphism. Then the projection of $\eta$ to $C(m+1)$ is surjective. Since the image of the three first maps in $((2.5 .2))$ is $A(m+1) \oplus B(m+1)$ we may conclude that the image of $\beta\left(\frac{m+1}{2}, \frac{m+1}{2}\right)$ is

$$
A(m+1) \oplus B(m+1) \oplus C(m+1)=H^{0}\left(\theta^{\otimes m+1}\right) .
$$

We proceed now to prove 2.3). Let $s_{1}, s_{2}$ satisfy $s_{1}+s_{2}=\frac{m+1}{2}$ and $s_{1}, s_{2} \geq 1$. Recall that

$$
H^{0}\left(\theta^{\otimes \frac{m+1}{2}}\right)=A\left(\frac{m+1}{2}\right) \oplus B\left(\frac{m+1}{2}\right) .
$$

However, if $s_{1}+s_{2}=\frac{m+1}{2}$ and $s_{1}, s_{2} \geq 1$, then $s_{1}, s_{2} \leq \frac{m-1}{2}$, hence $H^{0}\left(\theta^{\otimes s_{1}}\right)=$ $A\left(s_{1}\right)$ and $H^{0}\left(\theta^{\otimes s_{2}}\right)=A\left(s_{2}\right)$. Thus the map $\beta\left(s_{1}, s_{2}\right)$ is

$$
A\left(s_{1}\right) \otimes A\left(s_{2}\right) \longrightarrow A\left(\frac{m+1}{2}\right) \text {. }
$$


Thus the image of $\beta\left(s_{1}, s_{2}\right)$ is, for all $s_{1}+s_{2}=\frac{m+1}{2}, s_{1}, s_{2} \geq 1$, equal to $A\left(\frac{m+1}{2}\right)=H^{0}\left(\mathcal{O}_{\mathbf{P}^{1}}\left(\frac{m+1}{2} r\right)\right)$ which has codimension $\frac{r}{2}$ in $H^{0}\left(\theta^{\otimes \frac{m+1}{2}}\right)$.

Finally we prove 2.4). If $0 \leq s \leq \frac{m-1}{2}$ then $H^{0}\left(\theta^{\otimes s}\right)=A(s)$. Then if $s_{1}, s_{2} \geq 0$ and $s_{1}+s_{2} \leq \frac{m-1}{2}$, the map $\beta\left(s_{1}, s_{2}\right)$ is the multiplication map

$$
A\left(s_{1}\right) \otimes A\left(s_{2}\right) \longrightarrow A\left(s_{1}+s_{2}\right)
$$

which is surjective.

Now we prove 3$)$. Note that the vector subspace of $\left(R_{\theta}\right)_{n}$ generated by the elements of degree smaller than $n$ is the sum of the images of the maps $\beta\left(s_{1}, s_{2}\right)$ with $s_{1}, s_{2} \geq 1$ and $s_{1}+s_{2}=n$. We saw in 2.1) that the maps $\beta_{l}$ surject if $l<\frac{m-1}{2}$. Then the parts of $R_{\theta}$ of degree less than $\frac{m+1}{2}$ are generated by the elements of degree 1. 2.3) tells us that the elements of degree less than $\frac{m+1}{2}$ generates a subspace of $\left(R_{\theta}\right)_{\frac{m+1}{2}}$ of codimension $\frac{r}{2}$, henceforth we need $\frac{r}{2}$ linearly independent elements of degree $\frac{m+1}{2}$ to generate $\left(R_{\theta}\right)_{\frac{m+1}{2}}$. If $\frac{m+1}{2} \leq$ $l \leq m-1$, we saw also in 2.1) that the maps $\beta_{l}$ surject, therefore the parts of degree $\frac{m+1}{2}, \ldots, m$ are generated by the elements of $R_{\theta}$ of degree less than or equal to $\frac{m+1}{2}$. From 2.2) it follows that the map $\beta\left(\frac{m+1}{2}, \frac{m+1}{2}\right)$ surjects, therefore $\left(R_{\theta}\right)_{m+1}$ is also generated by the elements of $\left(R_{\theta}\right)_{\frac{m+1}{2}}$. Finally, if $l \geq m+1$ we saw in 2.1) that the map $\beta_{l}$ surjects, thus the parts of $R_{\theta}$ of degree greater than or equal to $m+2$ are generated by the elements of degree $m+1$. Summarizing the ring $R_{\theta}$ is generated by its elements of degree 1 and by $\frac{r}{2}$ elements of degree $\frac{m+1}{2}$.

Now we can prove Theorem 1 when the dimension of the variety of general type $X$ is odd. Precisely we prove

Theorem 2.6. Let $X$ be a smooth, pluriregular variety of general type of odd dimension $m \geq 3$ and let $Y \subset \mathbf{P}^{r+m-1}$ be an $m$-fold of minimal degree $r$. Assume that $K_{X}$ is base-point-free and let $\pi: X \longrightarrow Y$ be the canonical morphism of $X$. Assume that $\pi$ is generically finite morphism of degree 3 . Then the canonical ring of $X$ is generated by its part of degree 1 and $\frac{r}{2}$ generators in degree $\frac{m+1}{2}$.

Proof. Let $C$ be the intersection of $m-1$ general members of $\left|K_{X}\right|$. Then $C$ is a smooth and irreducible curve in $X$. Let $\theta=\left.K_{X}\right|_{C}$. By adjunction $\theta^{\otimes m}=K_{C}$. We set $\varphi=\left.\pi\right|_{C}$. Since $C$ has been taken general, we may, and actually do, 
choose $C$ so that $\varphi$ is finite onto a smooth rational normal curve $D$ of degree $r$ in $\mathbf{P}^{r}$.

As we have seen in the proof of Proposition 2.4, in order to find out the degrees of the generators of $R$, we only need to study the images of the maps $\alpha(s, t)$ for all $s, t \geq 1$. In fact, since many of them will happen to be surjective it will suffice, for our purpose, to study only some of them. More precisely the statement of the theorem is true if the following happens:

1) $H^{0}\left(K_{X}^{\otimes n}\right) \otimes H^{0}\left(K_{X}\right) \stackrel{\alpha_{n}}{\longrightarrow} H^{0}\left(K_{X}^{\otimes n+1}\right)$ surject for all $n \geq 0, n \neq \frac{m-1}{2}, m$;

2) $H^{0}\left(K_{X}^{\otimes \frac{m+1}{2}}\right) \otimes H^{0}\left(K_{X}^{\otimes \frac{m+1}{2}}\right) \stackrel{\alpha\left(\frac{m+1}{2}, \frac{m+1}{2}\right)}{\longrightarrow} H^{0}\left(K_{X}^{\otimes m+1}\right)$ surjects;

3) the images of

$$
H^{0}\left(K_{X}^{\otimes s_{1}}\right) \otimes H^{0}\left(K_{X}^{\otimes s_{2}}\right) \stackrel{\alpha\left(s_{1}, s_{2}\right)}{\longrightarrow} H^{0}\left(K_{X}^{\otimes \frac{m+1}{2}}\right)
$$

for all $s_{1}, s_{2} \geq 1, s_{1}+s_{2}=\frac{m+1}{2}$ are equal of codimension $\frac{r}{2}$.

Recall that $\varphi=\left.\pi\right|_{C}$ is induced by the complete series of $\theta$ and that $C, \theta$ and $\varphi$ satisfy the hypothesis of Proposition 2.4. Now to prove 1), 2) and 3) we will relate the maps appearing in 1),2) and 3) with the multiplication maps on $C$ studied in Proposition 2.4. To establish the relation between them we will use among other things Lemma 2.3. 1) and 2) follows directly from Lemma 2.3 (2) and Proposition 2.4 (2.1) and 2.4 (2.2). We now prove 3). By Proposition 2.4 (2.4) we know that the maps $\beta\left(s_{1}, s_{2}\right)$ are surjective if $s_{1}+s_{2} \leq \frac{m-1}{2}$. Let us know fix $s_{1}, s_{2} \geq 1$ such that $s_{1}+s_{2}=\frac{m+1}{2}$. Then by Lemma 2.3 (3), we know that the codimension of the image of $\alpha\left(s_{1}, s_{2}\right)$ in $H^{0}\left(K_{X}^{\otimes \frac{m+1}{2}}\right)$ is the same as the codimension of the image of $\beta\left(s_{1}, s_{2}\right)$ in $H^{0}\left(\theta^{\otimes \frac{m+1}{2}}\right)$. We also know by Lemma 2.3 (3) that the image of $\alpha\left(s_{1}, s_{2}\right)$ is the inverse image by the obvious map of restriction of sections

$$
H^{0}\left(K_{X}^{\otimes \frac{m+1}{2}}\right) \longrightarrow H^{0}\left(\theta^{\otimes \frac{m+1}{2}}\right)
$$

of the image of $\beta\left(s_{1}, s_{2}\right)$. Finally Proposition 2.4 (2.3) tells the image of $\beta\left(s_{1}, s_{2}\right)$ in $H^{0}\left(\theta^{\otimes \frac{m+1}{2}}\right)$ are the same for all $s_{1}, s_{2} \geq 1$ such that $s_{1}+s_{2}=\frac{m+1}{2}$ and have codimension $\frac{r}{2}$. Then all this implies that the images of $\alpha\left(s_{1}, s_{2}\right)$ are equal and of codimension $\frac{r}{2}$ in $H^{0}\left(K_{X}^{\otimes \frac{m+1}{2}}\right)$ for all $s_{1}, s_{2} \geq 1$ such that $s_{1}+s_{2}=\frac{m+1}{2}$.

Remark 2.7. Proposition 2.4 states explicitely that the ring $\bigoplus_{n \geq 0} H^{0}\left(C, \theta^{\otimes n}\right)$ has in each degree the same number of generators as the canonical ring of $X$, 
$\bigoplus_{n \geq 0} H^{0}\left(X, K_{X}^{\otimes n}\right)$. The way Theorem 2.6 is proved shows that the same is true also for the "intermediate" rings $\bigoplus_{n \geq 0} H^{0}\left(X_{m^{\prime}}, L_{m^{\prime}}^{\otimes n}\right), 2 \leq m^{\prime} \leq m-1$.

Knowing the generators of the canonical ring of $X$ tells us about the generators of the pluricanonical rings of $X$ and in particular, about which pulricanonical rings are generated in degree 1 . We state te result we obtain in this regard in the next Theorem. Before that we recall the following definition due to M. Green:

Definition 2.8. Let $L$ be a line bundle on a projective variety $X$. We say that $L$ satisfies property $N_{0}$ if $L$ is very ample and the image of $X$ by the embedding induced by $|L|$ is projectively normal.

Theorem 2.9. Let $X$ be a smooth, pluriregular variety of general type of odd dimension $m \geq 3$ and let $Y \subset \mathbf{P}^{r+m-1}$ be an $m$-fold of minimal degree $r$. Assume that $K_{X}$ is ample base-point-free and let $\pi: X \longrightarrow Y$ be the canonical morphism of $X$. Assume that $\pi$ has degree 3 . Then the line bundle $K_{X}^{\otimes n}$ satisfies property $N_{0}$ if and only if $n \geq \frac{m+1}{2}$.

Proof. Let $C$ be as in Theorem 2.6. We carry out the proof in several steps.

Step 1. $K_{X}^{\otimes n}$ does not satisfy property $N_{0}$ if $n<\frac{m+1}{2}$.

Indeed, if $n<\frac{m+1}{2}$, then

$$
H^{0}\left(K_{X}^{\otimes n}\right) \longrightarrow H^{0}\left(\left.K_{X}^{\otimes n}\right|_{C}\right)
$$

surjects by Lemma 2.2 and

$$
H^{0}\left(\left.K_{X}^{\otimes n}\right|_{C}\right)=H^{0}\left(\theta^{\otimes n}\right)=H^{0}\left(\mathcal{O}_{\mathbf{P}^{1}}(n r)\right)
$$

hence the $n$-pluricanonical morphism of $X$ maps $C$ to a rational normal curve, therefore $K_{X}^{\otimes n}$ is not very ample and does not satisfy property $N_{0}$.

Now to see that $K_{X}^{\otimes n}$ satisfies property $N_{0}$ for given $n \geq \frac{m+1}{2}$, it suffices to prove the surjectivity of the following maps:

$$
H^{0}\left(K_{X}^{\otimes l n}\right) \otimes H^{0}\left(K_{X}^{\otimes n}\right) \stackrel{\alpha(l n, n)}{\longrightarrow} H^{0}\left(K_{X}^{\otimes(l+1) n}\right), \text { for all } l \geq 1
$$

Step 2. $K_{X}^{\otimes n}$ satisfies property $N_{0}$ if $n=\frac{m+1}{2}$.

The map $\alpha\left(\frac{m+1}{2}, \frac{m+1}{2}\right)$ surjects as shown in 2) of the proof of Theorem 2.6. On the other hand, the surjectivity of $\alpha(\ln , n), l \geq 2$ and $n=\frac{m+1}{2}$ follows from 
the surjectivity of $\alpha_{n^{\prime}}$ for all $n^{\prime} \geq m+1$, which was also shown in 1) of the proof of Theorem 2.6.

Step 3. $K_{X}^{\otimes n}$ satisfies property $N_{0}$ if $\frac{m+1}{2}<n \leq m$. We first show that $\alpha(n, n)$ surjects. Since $\alpha\left(\frac{m+1}{2}, \frac{m+1}{2}\right)$ surjects and so do the maps $\alpha_{n^{\prime}}$ if $n^{\prime} \geq m+1$, the following multiplication map

$$
H^{0}\left(K^{\otimes \frac{m+1}{2}}\right) \otimes H^{0}\left(K_{X}^{\otimes\left(n-\frac{m+1}{2}\right)}\right) \otimes H^{0}\left(K^{\otimes \frac{m+1}{2}}\right) \otimes H^{0}\left(K_{X}^{\otimes\left(n-\frac{m+1}{2}\right)}\right) \stackrel{\gamma}{\rightarrow} H^{0}\left(K_{X}^{\otimes 2 n}\right)
$$

also surjects. On the other hand $\gamma$ factorizes through $\alpha(n, n)$, which is therefore surjective.

Finally the surjectivity of the maps $\alpha(\ln , n)$ for all $l \geq 2$ and $\frac{m+1}{2}<n \leq m$ follows from the surjectivity of $\alpha_{n^{\prime}}$ for all $n^{\prime} \geq m+1$.

Step 4. $K_{X}^{\otimes n}$ satisfies property $N_{0}$ if $n \geq m+1$. The surjectivity of the maps $\alpha_{n^{\prime}}$ for all $n^{\prime} \geq m+1$ implies the surjectivity of the maps $\alpha(\ln , n)$ for all $l \geq 1$ and $n \geq m+1$.

Now we proceed to prove Theorem 1 when the dimension of $X$ is even. As we did in the odd dimensional case, we need study first multiplication maps on a curve $C$, which is a complete intersection on $X$. Thus we prove a result analogous to Proposition 2.4.

Proposition 2.10. Let $C$ be a smooth curve, let $\theta$ be an ample and base-pointfree line bundle on $C$ and let $m$ be an even natural number such that $\theta^{\otimes m}=K_{C}$. Let $\varphi: C \longrightarrow Y$ be the morphism induced by $|\theta|$ and assume that the degree of $\varphi$ is 3 and that $Y$ is a (smooth) rational normal curve of degree $r$. Then

$$
\left.\varphi_{*}\left(\mathcal{O}_{C}\right)=\mathcal{O}_{\mathbf{P}^{1}} \oplus \mathcal{O}_{\mathbf{P}^{1}}\left(-\frac{m r+2}{2}\right) \oplus \mathcal{O}_{\mathbf{P}^{1}}(-m r-2)\right)
$$

1.2) The map $\varphi$ is induced by the complete linear series of $\theta$.

2.1) The map $\beta_{n}$ surjects if $n \neq \frac{m}{2}, m, m+1$; if $n=m+1$ and $r \geq 2$; and if $n=m$ and $r=1$.

2.2) The images of the maps $\beta\left(s_{1}, s_{2}\right)$ are equal and of codimension $r$ in $H^{0}\left(\theta^{\otimes \frac{m+2}{2}}\right)$ if $s_{1}, s_{2} \geq 1$ and $s_{1}+s_{2}=\frac{m+2}{2}$.

2.3) The images of the maps $\beta\left(s_{1}, s_{2}\right)$ are equal and of codimension $r-1$ in $H^{0}\left(\theta^{\otimes m+1}\right)$ if $s_{1}, s_{2} \geq 1$ and $s_{1}+s_{2}=m+1$.

2.4) The $\operatorname{map} \beta\left(\frac{m+2}{2}, \frac{m+2}{2}\right)$ surjects.

2.5) The map $\beta\left(s_{1}, s_{2}\right)$ surjects if $s_{1}, s_{2} \geq 0$ and $s_{1}+s_{2} \leq \frac{m}{2}$. 
2.6) The map $\beta\left(s_{1}, s_{2}\right)$ surjects if $s_{1}, s_{2} \geq 0, s_{1} \geq \frac{m+2}{2}$, and $\frac{m+2}{2} \leq s_{1}+s_{2} \leq m$.

3) The ring $R_{\theta}=\bigoplus_{s=1}^{\infty}\left(R_{\theta}\right)_{s}$, where $\left(R_{\theta}\right)_{s}=H^{0}\left(\theta^{\otimes s}\right)$, is generated by its part of degree $r$ generators in degree $\frac{m+2}{2}$ and $r-1$ generators in degree $m+1$.

Proof. The proof of 1.1) and 1.2) is exactly like the proof of parts 1.1) and 1.2) of Proposition 2.4. The proof of 2) is also like the proof of part 2) of Proposition 2.4. Here we will only highlight the differences. As we saw in the proof of Proposition 2.4 (2), the key to know the images of the maps $\beta\left(s_{1}, s_{2}\right)$ is the decomposition of $H^{0}\left(\theta^{\otimes s_{1}}\right)$ and $H^{0}\left(\theta^{\otimes s_{2}}\right)$ as sum of blocks $A\left(s_{1}\right) \oplus B\left(s_{1}\right) \oplus$ $C\left(s_{1}\right)$ and $A\left(s_{2}\right) \oplus B\left(s_{2}\right) \oplus C\left(s_{2}\right)$, i.e., the crucial information is to know which ones among $A\left(s_{1}\right), B\left(s_{1}\right), C\left(s_{1}\right), A\left(s_{2}\right), B\left(s_{2}\right), C\left(s_{2}\right)$ are zero. This information is obtained from 1). If $0 \leq n \leq \frac{m}{2}$, then $H^{0}\left(\theta^{\otimes n}\right)=A(n)$. Thus, the map $\beta_{n}$ surjects if $0 \leq n \leq \frac{m-2}{2}$. If $\frac{m+2}{2} \leq n \leq m, H^{0}\left(\theta^{\otimes n}\right)=A(n) \oplus B(n)$, hence the map $\beta_{n}$ also surjects if $\frac{m+2}{2} \leq n \leq m-1$. If $n \geq m+2, H^{0}\left(\theta^{\otimes n}\right)=A(n) \oplus$ $B(n) \oplus C(n)$, then the map $\beta_{n}$ surjects if $n \geq m+2$. If $r=1, H^{0}\left(\theta^{\otimes m+1}\right)=$ $A(m+1) \oplus B(m+1)$, thus $\beta_{m}$ surjects if $r=1$. If $r>1$, then $H^{0}\left(\theta^{\otimes m+1}\right)=$ $A(m+1) \oplus B(m+1) \oplus C(m+1)$, therefore $\beta_{m+1}$ surjects if $r>1$. This proves 2.1). Now we prove 2.2). Recall that if $0 \leq n \leq \frac{m}{2}$, then $H^{0}\left(\theta^{\otimes n}\right)=A(n)$. On the other hand $H^{0}\left(\theta^{\otimes \frac{m+2}{2}}\right)=A\left(\frac{m+2}{2}\right) \oplus B\left(\frac{m+2}{2}\right)$. Since $B\left(\frac{m+2}{2}\right)=H^{0}\left(\mathcal{O}_{\mathbf{P}^{1}}(r-1)\right)$, then, if $s_{1}, s_{2} \geq 0$ and $s_{1}+s_{2}=\frac{m+2}{2}$ the image of the maps $\beta\left(s_{1}, s_{2}\right)$ are equal to $A\left(\frac{m+2}{2}\right)$, which has codimension $r$ in $H^{0}\left(\theta^{\otimes \frac{m+2}{2}}\right)$. To prove 2.3$)$ recall that if $\frac{m+2}{2} \leq n \leq m, H^{0}\left(\theta^{\otimes n}\right)=A(n) \oplus B(n)$ and that $H^{0}\left(\theta^{\otimes m+1}\right)=A(m+1) \oplus$ $B(m+1) \oplus C(m+1)$. Thus, if $s_{1}, s_{2} \geq 0$ and $s_{1}+s_{2}=m+1$, then either $s_{1} \geq \frac{m+1}{2}$ or $s_{2} \geq \frac{m+1}{2}$. Let us say that $s_{1} \geq \frac{m+1}{2}$, then $s_{2} \leq \frac{m+1}{2}$. Then the image of the maps $\beta\left(s_{1}, s_{2}\right)$ is $A(m+1) \oplus B(m+1)$. Since $C(m+1)=H^{0}\left(\mathcal{O}_{\mathbf{P}^{1}}(r-2)\right)$, the codimension of the image of $\beta\left(s_{1}, s_{2}\right)$ in $H^{0}\left(\theta^{\otimes m+1}\right)$ is $r-1$. Part 2.4) follows from the fact that $H^{0}\left(\theta^{\otimes \frac{m+2}{2}}\right)=A\left(\frac{m+2}{2}\right) \oplus B\left(\frac{m+2}{2}\right)$ arguing as in the proof of Proposition 2.4 (2.2). Part 2.5) follows from the fact that $H^{0}\left(\theta^{\otimes n}\right)=A(n)$ if $0 \leq n \leq \frac{m}{2}$. Now we prove 2.6). Recall that if $\frac{m+2}{2} \leq n \leq m$, then $H^{0}\left(\theta^{\otimes n}\right)=$ $A(n) \oplus B(n)$ and that if $0 \leq n \leq \frac{m}{2}$, then $H^{0}\left(\theta^{\otimes n}\right)=A(n)$. This implies 2.6).

Finally 3) follows from 2.1), 2.2), 2.3) and 2.4).

Now from the previous Proposition 2.10 and using Lemma 2.2 and Lemma 2.3 we can prove Theorem 1 for the even dimensional case:

Theorem 2.11. Let $X$ be a smooth, pluriregular variety of general type of even dimension $m$ with $K_{X}$ base-point-free. Assume that the canonical morphism $\pi$ 
is generically finite of degree 3 onto a variety $Y$ of minimal degree $r$. Then the canonical ring of $X$ is generated by its elements of degree $1, r$ generators in degree $\frac{m+2}{2}$ and $r-1$ generators in degree $m+1$.

Sketch of proof. The proof uses the same ideas as the proof of Theorem 2.6 so we will just outline the key steps. As in Theorem 2.6 we need to know the images of the maps $\alpha\left(s_{1}, s_{2}\right)$. Precisely the result follows from the following claims:

1) The map $\alpha_{n}$ surjects if $1 \leq n \leq \frac{m-2}{2}$.

2) The images of the maps $\alpha(s, t)$, when $s+t=\frac{m+2}{2}$ and $s, t \geq 1$ are equal and of codimension $r$ in $H^{0}\left(K_{X}^{\otimes \frac{m+2}{2}}\right)$.

3) The map $\alpha_{n}$ surjects if $\frac{m+2}{2} \leq n \leq m-1$.

4) The images of the maps $\alpha(s, t)$, when $s+r=m+1, s, t \geq 1$ are equal and of codimension $r-1$ in $H^{0}\left(K_{X}^{\otimes m+1}\right)$.

5) The map $\alpha\left(\frac{m+2}{2}, \frac{m+2}{2}\right)$ surjects if $r=1$.

6) The map $\alpha_{m+1}$ surjects if $r \geq 2$.

7) The map $\alpha_{n}$ surjects if $n \geq m+2$.

Before we prove the above claims, we will show how they imply the result. Claim 1) implies that the part of $R$ of degree less than or equal to $\frac{m}{2}$ is generated in degree 1. Claim 2) implies that the subspace of the part of degree $\frac{m+2}{2}$ generated in lower degree has codimension $r$, hence, in order to generate $R, r$ generators of degree $\frac{m+2}{2}$ are needed. Claim 1) and 3) imply that the part of $R$ of degree less than or equal to $m$ is generated in degree 1 and $\frac{m+1}{2}$. By the same argument as before, Claim 4) implies that, in order to generate $R$, we need $r-1$ linearly independent elements of degree $m+1$. Claims 5) and 6) imply that $R_{m+2}$ is generated in degree $\frac{m+2}{2}$ or lower. Finally, Claim 7) proves that the part of $R$ of degree greater than $m+2$ is generated in degree $m+2$ or lower. Summarizing, the above claims show that $R$ is generated by its part of degree 1 , by $r$ linearly independent elements in degree $\frac{m+2}{2}$ and by $r-1$ linearly independent elements in degree $m+1$.

Now we proceed to prove claims 1) to 7 ). The proof goes like the proof of Theorem 2.6. Thus Claims 1), 3), 6) and 7) follow from Proposition 2.10 (2.1) and Lemma 2.3 (2). The proof of Claim 2) follows from Proposition 2.10 (2.2) and Proposition 2.10 (2.5), and from Lemma 2.3 (3), arguing like for the proof the 3 ) in the proof of Theorem 2.6. Likewise Claim 4) follows from Proposition 2.10 (2.3) and 2.10 (2.6) and from Lemma 2.3(3). Finally Claim 5) follows from Proposition 2.10 (2.3), (2.4) and 2.10 (2.6) and from Lemma 2.3 (3). 
Remark 2.12. Proposition 2.10 states explicitely that the ring $\bigoplus_{n \geq 0} H^{0}\left(C, \theta^{\otimes n}\right)$ has in each degree the same number of generators as $\bigoplus_{n \geq 0} H^{0}\left(X, K_{X}^{\otimes n}\right)$. The way Theorem 2.11 is proven shows that the same is true for the "intermediate" rings $\bigoplus_{n \geq 0} H^{0}\left(X_{m^{\prime}}, L_{m^{\prime}}^{\otimes n}\right), 2 \leq m^{\prime} \leq m-1$.

We find now sufficient conditions for $K_{X}^{\otimes n}$ to satisfy property $N_{0}$ :

Theorem 2.13. Let $X$ be a smooth, pluriregular variety of general type of even dimension $m$ with ample and base-point-free canonical bundle. Assume that the canonical morphism has degree 3 and maps $X$ onto a variety $Y$ of minimal degree $r$.

1) If $r=1$, then the line bundle $K_{X}^{\otimes n}$ satisfies propety $N_{0}$ if and only if $n \geq \frac{m+2}{2}$.

2) If $r>1$, then the line bundle $K_{X}^{\otimes n}$ fails to satisfy property $N_{0}$ if $n \leq \frac{m}{2}$ and satisfies property $N_{0}$ if $n \geq m+1$.

Proof. The proof of 1) follows steps similar to those of the proof of Theorem 2.9. Step 1. $K_{X}^{\otimes n}$ does not satisfy property $N_{0}$ if $n \leq \frac{m}{2}$.

Step 2. $K_{X}^{\otimes n}$ satisfies property $N_{0}$ if $n=\frac{m+2}{2}$.

Step 3. $K_{X}^{\otimes n}$ satisfies property $N_{0}$ if $\frac{m+4}{2} \leq n \leq m+1$.

Step 4. $K_{X}^{\otimes n}$ satisfies property $N_{0}$ if $n \geq m+2$. Step 1 is true by the same reason as Step 1 of the proof of Theorem 2.9. The other steps are proven like in Theorem 2.9 and the key facts to use are the surjectivity of $\alpha\left(\frac{m+2}{2}, \frac{m+2}{2}\right)$ if $r=1$ and the surjectivity of $\alpha_{n^{\prime}}$ for all $n^{\prime} \geq m+2$. This was shown in 5) and 7) of the proof of Theorem 2.11. We leave the rest of the details to the reader.

The proof of 2) goes along the same lines. The fact that the line bundle $K_{X}^{\otimes n}$ fails to satisfy property $N_{0}$ if $n \leq \frac{m}{2}$ is proven like Step 1 of Theorem 2.9. The fact that $K_{X}^{\otimes n}$ satisfies property $N_{0}$ if $n \geq m+1$ follows from the surjectivity of $\alpha_{n^{\prime}}$ for all $n^{\prime} \geq m+1$ and $r>1$.

Comparing the previous result with Theorem 2.9 we realize that in the even dimensional case the following question is left open:

Question 2.14. Let $X$ be a smooth variety of general type and dimension $m$ even and let $X \stackrel{\pi}{\longrightarrow} Y$ be a generically finite, canonically induced, degree 3 cover of a variety $Y$ of minimal degree $r>1$. Does $K_{X}^{\otimes n}$ satisfy property $N_{0}$ if $\frac{m+2}{2} \leq n \leq m$ ?

The reason why this question cannot be addressed with the present arguments is that we cannot decide whether the map $\alpha\left(\frac{m+2}{2}, \frac{m+2}{2}\right)$ surjects. The map 
$\beta\left(\frac{m+2}{2}, \frac{m+2}{2}\right)$ does surject, as stated in Proposition $2.10(2.4)$, but if we were to use Lemma 2.3 to deduce from the surjectivity of $\beta\left(\frac{m+2}{2}, \frac{m+2}{2}\right)$ the surjectivity of $\alpha\left(\frac{m+2}{2}, \frac{m+2}{2}\right)$, we would also need the surjectivity of $\beta\left(\frac{m+2}{2}, \frac{m}{2}\right)$. The latter map is however non surjective (cf. Proposition $2.10(2.3)$ ).

\section{ON THE STRUCTURE OF THE CANONICAL MORPHISM}

In the previous section we studied the canonical ring of pluriregular varieties of general type $X$ which were triple canonical covers of varieties of minimal degree. In this section we relax our hypothesis on $X$ and $\pi$ and study the structure of the canonical morphism $X \stackrel{\pi}{\rightarrow} Y$. This study also tells us about the structure of $X$ and $Y$. We do not not assume a priori $X$ to be pluriregular or even regular, neither do we assume $\pi$ to be induced by the complete canonical series of $X$. In fact we start only with a base-point-free canonical subseries (we will see later, cf. Theorem 3.3, that assuming $\pi$ to be flat is sufficient for $X$ to be as the varieties studied in Section 2, that is, pluriregular and with $\pi$ induced by the complete canonical series). Despite these weaker hypothesis, in the next theorem we are able to obtain interesting information relating the degree of $Y$ and the dimension of $X$ :

Theorem 3.1. Let $X$ be a smooth variety of general type of dimension $m \geq 2$ and let $Y \subset \mathbf{P}^{r+m-1}$ be an m-fold of minimal degree. Assume that $K_{X}$ is base-point-free and let $W$ be a sublinear series of $\left|K_{X}\right|$ without base points. Let $\pi: X \longrightarrow Y$ be the morphism induced by $W$. Assume that $\pi$ is generically finite of degree 3 . Then

1) If $m$ is odd, then the degree $r$ of $Y$ is even (in particular $Y$ is not linear $\mathbf{P}^{m}$ )

2) If $h^{1}\left(\mathcal{O}_{X}\right)=0$, then $\pi$ is induced by the complete canonical series of $X$.

Proof. Since $W$ has no base points, we may choose $m-1$ general members of $W$ so that its intersection $C$ is a smooth and irreducible curve in $X$ and so that $\varphi=\left.\pi\right|_{C}$ is finite onto a smooth rational normal curve $D$ of degree $r$ in $\mathbf{P}^{r}$. Let $\theta=\left.K_{X}\right|_{C}$. By adjunction $\theta^{\otimes m}=K_{C}$. Now, if $m$ is odd, then $C, \theta$ and $\varphi$ satisfy the hypothesis of Proposition 2.4. Then according to Proposition 2.4 (1.1), $r$ is even. On the other hand, $C, \theta$ and $\varphi$ satisfy the hypothesis of Proposition 2.4 if $m$ is odd, and the hypothesis of Proposition 2.10 if $m$ is even. Hence, since $m \geq 2$, according to Proposition 2.4 (1.2) and Proposition 2.10 (1.2), $\varphi$ is induced by the complete linear series $|\theta|$. Since $H^{1}\left(\mathcal{O}_{X}\right)=0$, by Kawamata-Viehweg vanishing we have also $H^{1}\left(\mathcal{O}_{X_{m-1}}\right)=\cdots=H^{1}\left(\mathcal{O}_{X_{2}}\right)=0$. 
Then the fact that $\varphi$ is induced by the complete $|\theta|$ implies that $\pi$ is induced by the complete linear series of the canonical of $X$. This proves 2 ).

Theorem 3.1 tells us among other things that a variety of minimal degree $Y$ with degree an even integer, cannot occur as the image of a canonical morphism $X \stackrel{\pi}{\longrightarrow} Y$ of degree 3 if the dimension of $X$ is odd. In the next result we further eliminate possible targets of $\pi$. Applied to the setting of Section 2, in which $Y$ was a variety of minimal degree and $\pi$ has degree 3, Theorem 3.2 tells us that there are no even dimensional canonical, generically triple covers of smooth rational normal scrolls. Theorem 3.2 is more general since it considers generically finite canonical morphisms of arbitrary odd degree and smooth scrolls fibered not necessarily over $\mathbf{P}^{1}$.

Theorem 3.2. Let $X$ be a smooth variety of general type of dimension $m$ with base-point-free canonical bundle $X$. Let $W$ be a linear subseries of $\left|K_{X}\right|$ without base points and let $X \stackrel{\pi}{\rightarrow} Y$ be the morphism induced by $W$. Assume that $\pi$ is generically finite of degree $n$ and that $Y$ is a scroll. Then either $n$ is even or $m$ is odd. In particular, if $n=3, m$ is odd.

Proof. Let $G$ be a fiber of Y. Let $H$ be the class of the hyperplane section of Y. Let $g$ be a straight line in $G$. Then $g=G \cdot H^{(m-2)}$, i.e, $\mathrm{g}$ is the complete intersection on $Y$ of $G$ and $m-2$ suitable hyperplane sections. Likewise, $\pi^{*} g$ is the complete intersection on $X$ of the pullback by $\pi$ of $m-2$ suitable hyperplane sections and $\pi^{*} G$. We choose the $m-2$ hyperplane sections suitably so that, by Bertini, the intersection of the pullback by $\pi$ of $m-2$ of them is a smooth irreducible surface $X^{\prime}$. The image of $X^{\prime}$ by $\pi$ is a surface $Y$, which is also a smooth scroll and has dimension 2. Applying adjunction recursively we see that $K_{X^{\prime}}$ is the restriction of $K_{X}+(m-2) \pi^{*}(H)$ to $X^{\prime}$. Then the sectional genus of $\pi^{*} G$ is $t=\left(K_{X^{\prime}}+\pi^{*} g\right) \cdot \pi^{*} g$. Now we know that $K_{X}=\pi^{*} H$, so we have that

$t=\left[K_{X}+(m-2) \pi^{*} H+\pi^{*} G\right] \cdot \pi^{*} g=\left[(m-1) \pi^{*} H+\pi^{*} G\right] \cdot \pi^{*} g=(m-1) n$.

Thus, if $m$ is even, $m-1$ is odd, so $n$ has to be even. And if $n$ is odd, $m-1$ has to be even, hence $m$ has to be odd.

The two previous results eliminate several possibilities for the target of the morphism $X \stackrel{\pi}{\rightarrow} Y$. We end the section characterizing, under the stronger hypothesis of $\pi$ being flat, the targets that actually do occur. We see this in the next theorem, where we give the list of the only possible targets. In 
Proposition 3.4, we show that all the possibilities allowed by Theorem 3.3 do exist. Theorem 3.3 also shows that a variety of general type which admits a flat, triple canonical morphism onto a variety of minimal degree is necessarily pluriregular. The theorem below also describes some features of the morphism $\pi$.

Theorem 3.3. Let $X$ be a smooth variety of general type of dimension $m \geq 2$. Assume that $K_{X}$ is ample and base-point-free. Let $W$ be a linear subseries of $\left|K_{X}\right|$ without base points. Let $\pi$ be the morphism induced by $W$. Assume that $\pi$ is flat and has degree 3 . Let $Y=\pi(X)$ be a variety of minimal degree $r$. Then $Y$ is one of the following:

1) $\mathbf{P}^{m}$ with $m \geq 2$ even.

2) A smooth rational normal scroll of odd dimension $m \geq 3$ and even degree $r$. In this case $X$ is fibered over $\mathbf{P}^{1}$, with general fiber a smooth variety of general type of dimension $m-1$. The restriction of $\pi$ to the general fiber is finite and flat, induced by the complete canonical series, and maps onto $\mathbf{P}^{m-1}$, fitting therefore in case 1) above.

3) A smooth quadric hypersurface of odd dimension $m \geq 3$.

In addition, $\pi$ is in fact induced by the complete canonical linear series of $X$; $\pi_{*} \mathcal{O}_{X}=\mathcal{O}_{Y} \oplus L^{-1} \oplus L^{-2}$ for a line bundle $L$ such that $L^{-2}$ is not effective; $K_{Y}=L^{-2}(1)$ and $H^{1}\left(\mathcal{O}_{X}\right)=\cdots=H^{m+1}\left(\mathcal{O}_{X}\right)=0$.

Proof. Since $\pi$ is flat and $X$ is smooth, $Y$ is also smooth. Thus $Y$ must be one of the following:

a) $\mathbf{P}^{m}$;

b) a smooth rational normal scroll;

c) a smooth quadric hypersurface.

Since $\pi$ is finite of degree 3 and flat, $\pi_{*} \mathcal{O}_{X}=\mathcal{O}_{Y} \oplus E$, where $E$ is a locally free sheaf of rank 2 (cf. $[\mathrm{HM}]$ ). Assume $E$ does not decompose. Then since $\pi$ is induced by a canonical subseries, $K_{X}=\pi^{*}\left(\mathcal{O}_{Y}(1)\right)$. Hence by projection formula, $\pi_{*} K_{X}=\mathcal{O}_{Y}(1) \oplus E(1)$. On the other hand, by relative duality $\pi_{*} K_{X}=$ $K_{Y} \oplus\left(E^{*} \otimes K_{Y}\right)$, hence $K_{Y}=\mathcal{O}_{Y}(1)$ which is a contradiction. Then $E=$ $L_{1}^{-1} \oplus L_{2}^{-1}$, for line bundles $L_{1}$ and $L_{2}$ such that neither $L_{1}^{-1}$ nor $L_{2}^{-2}$ has sections, for $X$ is connected. Furthermore

$$
\pi_{*} K_{X}=\mathcal{O}_{Y}(1) \oplus L_{1}^{-1}(1) \oplus L_{2}^{-1}(1)
$$

on the one hand and on the other hand,

$$
\pi_{*} K_{X}=K_{Y} \oplus\left(K_{Y} \otimes L_{1}\right) \oplus\left(K_{Y} \otimes L_{2}\right)
$$


We have already noticed that $K_{Y}$ cannot be $\mathcal{O}_{Y}(1)$. Then either $K_{Y}=L_{1}^{-1}(1)$ or $K_{Y}=L_{2}^{-1}(1)$. We see first what happens if $K_{Y}=L_{1}^{-1}(1)$. In this case $K_{Y} \otimes L_{1}$ equals either $\mathcal{O}_{Y}(1)$ or $L_{2}^{-1}(1)$. The latter leads to a contradiction. On the other hand, if $K_{Y}=L_{1}^{-1}(1)$ and $K_{Y} \otimes L_{1}=\mathcal{O}_{Y}(1)$, then $L_{1}=L_{2}^{\otimes 2}$ and $K_{Y}=L_{2}^{-2}(1)$. The case $K_{Y}=L_{2}^{-1}(1)$ is analogous, yielding $L_{2}=L_{1}^{\otimes 2}$ and $K_{Y}=L_{1}^{-2}(1)$. Thus we have shown that

$$
\text { (3.3.1) } \pi_{*} \mathcal{O}_{X}=\mathcal{O}_{Y} \oplus L^{-1} \oplus L^{-2},
$$

for a line bundle $L$ such that $L^{-2}$ is not effective. Moreover, $\pi_{*} K_{X}=\mathcal{O}_{Y}(1) \oplus$ $L^{-1}(1) \oplus L^{-2}(1)$ and

$$
\text { (3.3.2) } K_{Y}=L^{-2}(1)
$$

Now we study further restrictions on 1), 2) and 3). Let $Y$ be first $\mathbf{P}^{m}$. Since $K_{Y}=\mathcal{O}_{\mathbf{P}^{m}}(-m-1)$, by (3.3.2) $m$ has to be even. Let $Y$ be now a rational normal scroll. Let us denote by $H$ the divisor class of its hyperplane section and by $F$ the class of a fiber. Let $r$ be the degree of $Y$. Then $K_{Y}=-m H+(r-2) F$. Again by (3.3.2) $r$ has to be even and $m$ has to be odd. The only case left is when $Y$ is a smooth quadric hypersurface of dimension greater than 2. By Lefschetz Theorem, $\operatorname{Pic} Y=\mathbf{Z}$ and is generated by the hyperplane section $Y$. In addition $K_{Y}=\mathcal{O}_{Y}(-m)$ by adjunction. Hence by (3.3.2) $m$ is odd.

Since $L^{-2}(1)=K_{Y}$ and by projection formula

$$
H^{0}\left(\pi_{*} \pi^{*}\left(\mathcal{O}_{Y}(1)\right)=H^{0}\left(\mathcal{O}_{Y}(1)\right) \oplus H^{0}\left(L^{-1}(1)\right) \oplus H^{0}\left(L^{-2}(1)\right.\right.
$$

checking for each case a), b) c) we see that $H^{0}\left(\pi_{*} \pi^{*}\left(\mathcal{O}_{Y}(1)\right)=H^{0}\left(\mathcal{O}_{Y}(1)\right)\right.$, hence $\pi$ is in fact induced by the complete canonical series of $X$. To further study the structure of $\pi$ in case 2), let us denote by $G$ the inverse image of a general fiber $F$ of $Y$. We have the following exact sequence:

$$
0 \longrightarrow H^{0}\left(K_{X}(-G)\right) \longrightarrow H^{0}\left(K_{X}\right) \longrightarrow H^{0}\left(K_{X} \otimes \mathcal{O}_{G}\right) \longrightarrow H^{1}\left(K_{X}(-G)\right) .
$$

Since $\pi$ is finite, and $K_{X}(-G)=\pi^{*}\left(\mathcal{O}_{Y}(H-F)\right)$,

$$
H^{1}\left(K_{X}(-G)\right)=H^{1}\left(\mathcal{O}_{Y}(H-F) \oplus L^{-1}(H-F) \oplus L^{-2}(H-F)\right) .
$$

Since $L^{-1}(H-F)=\mathcal{O}_{Y}\left(\frac{-m+1}{2} H+\frac{r-4}{2} F\right)$ and $L^{-2}(H-F)=\mathcal{O}_{Y}(-m H+$ $(r-3) F)$, the first cohomology group of the three line bundle vanishes. Hence 
the complete linear series $\left|K_{X}\right|$ restricts to the complete linear series $K_{G}$, which maps onto $F=\mathbf{P}^{m-1}$. $G$ is smooth by Bertini, and since $K_{X}$ is ample and base-point-free, $G$ must be connected. The finiteness of the morphism from $G$ to $F$ and the fact that its degree is 3 are clear. To see the flatness it suffices to see that $\pi_{*}\left(\mathcal{O}_{X}\right) \otimes \mathcal{O}_{G}=\left(\left.\pi\right|_{G}\right)_{*}\left(\mathcal{O}_{G}\right)$.

Finally, to see the vanishings of cohomology we will deal case by case. Recall that $L^{-1}=\mathcal{O}_{Y}\left(\frac{1}{2}\left(K_{Y}-H\right)\right)$ and that $\pi_{*}\left(\mathcal{O}_{X}\right)=\mathcal{O}_{Y} \oplus L^{-1} \oplus L^{-2}$. If $Y=\mathbf{P}^{m}$, then $H^{i}\left(\mathcal{O}_{X}\right)=0$ for all $1 \leq i \leq m-1$, since intermediate cohomology of line bundles on $Y$ vanishes. If $Y$ is a smooth rational normal scroll, then

$$
\begin{aligned}
H^{i}\left(\mathcal{O}_{X}\right)= & H^{i}\left(\mathcal{O}_{Y}\right) \oplus H^{i}\left(\mathcal{O}_{Y}\left(-\frac{1}{2}(m+1) H+\frac{1}{2}(r-2) F\right)\right) \\
& \oplus H^{i}\left(\mathcal{O}_{Y}(-(m+1) H+(r-2) F)\right)=0
\end{aligned}
$$

for all $1 \leq i \leq m-1$. The latter can be seen using Serre duality and pushing down to $\mathbf{P}^{1}$. Now, for $Y$ smooth quadric hypersurface of dimension $m$ we have the sequence

$$
0 \longrightarrow \mathcal{O}_{\mathbf{P}^{m+1}}(-2) \longrightarrow \mathcal{O}_{\mathbf{P}^{m+1}} \longrightarrow \mathcal{O}_{Y} \longrightarrow 0
$$

so the vanishing of $H^{i}\left(\mathcal{O}_{Y}\right)$ for all $1 \leq i \leq m-1$ follows from the vanishing of the intermediate cohomology of line bundles on projective space.

Finally we show in the following proposition that all the possible varieties of minimal degree allowed by Theorem 3.3 do actually occur. Note also that, by the previous theorem, the varieties of general type constructed in the next proposition are pluriregular and satisfy therefore the hypothesis of Theorem 1.

\section{Proposition 3.4.}

a) There exist smooth varieties of general type $X$ with base-point-free canonical bundle $K_{X}$ and

cyclic triple covers $X \longrightarrow \mathbf{P}^{m}$ induced by the canonical morphism of $X$ if and only if $m$ is even.

b) Let $m \geq 3$ and let $Q \subset \mathbf{P}^{m+1}$ be a smooth hyperquadric of dimension $m$. There exist smooth varieties of general type $X$ with base-point-free canonical bundle $K_{X}$ and cyclic triple covers $X \longrightarrow Q$ induced by the canonical morphism of $X$ if and only if $m$ is odd.

c) Let $m \geq 3$. There exist smooth rational normal scrolls $S$ of dimension $m$ and degree $r$, smooth varieties of general type $X$ with base-point-free canonical bundle $K_{X}$ and cyclic triple covers $X \longrightarrow S$ induced by the canonical morphism of $X$ if and only if $m$ is odd and $r$ is even. 
Proof. Theorem 3.3 takes care of the "only if" part of a), b) and c). To construct examples of triple covers on $\mathbf{P}^{m}, m$ even, take the triple cyclic cover $X \stackrel{\pi}{\rightarrow} \mathbf{P}^{m}$ ramified along a smooth divisor of degree $\frac{3(m+2)}{2}$. In such case,

$$
K_{X}=\pi^{*}\left(K_{\mathbf{P}^{m}} \otimes \mathcal{O}_{\mathbf{P}^{m}}(m+2)\right)=\pi^{*} \mathcal{O}_{\mathbf{P}^{m}}(1) .
$$

Moreover

$H^{0}\left(K_{X}\right)=H^{0}\left(\mathcal{O}_{\mathbf{P}^{m}}(1)\right) \oplus H^{0}\left(\mathcal{O}_{\mathbf{P}^{m}}\left(-\frac{m}{2}\right)\right) \oplus H^{0}\left(\mathcal{O}_{\mathbf{P}^{m}}(-m-1)\right)=H^{0}\left(\mathcal{O}_{\mathbf{P}^{m}}(1)\right)$,

hence $\pi$ is induced by the complete canonical series of $X$. Analogously, to construct examples of triple cyclic covers of a hyperquadric $Q$ of odd dimension $m \geq 3$ we take the cover $X \stackrel{\pi}{\rightarrow} Q$ ramified along a smooth divisor in $Q$, complete intersection of a hypersurface of $\mathbf{P}^{m+1}$ of degree $\frac{3(m+1)}{2}$ and $Q$. Finally, to construct examples of triple cyclic covers of smooth rational normal scrolls of odd dimension $m \geq 3$ and even degree $r$, consider a smooth rational scroll $S$ possessing smooth divisors linearly equivalent to $\frac{m+1}{2} H-\frac{r-2}{2} F$, where $H$ is the hyperplane class of $S$ and $F$ is the class of a fiber. Then we take the cyclic triple cover $X \stackrel{\pi}{\longrightarrow} S$ ramified along a smooth divisor linearly equivalent to $\frac{m+1}{2} H-\frac{r-2}{2} F$.

Remark 3.5 Theorem 1 and Proposition 3.4 show that if $m \geq 3$, there does not exist a converse to the theorem of M. Green (cf. [G]) which says that if $X$ is a smooth, pluriregular variety of general type with base-point-free canonical bundle, then the canonical ring of $X$ is generated in degree less than or equal to $m$ if the image $Y$ of the canonical morphism $\pi$ of $X$ is not a variety of minimal degree. Indeed, the canonical ring of the varieties constructed in Proposition 3.4 are generated in degree less than or equal to $\frac{m+1}{2}$ if $m$ is odd and $\frac{m+2}{2}$ if $m$ is even. Therefore, $Y$ not being a canonical degree is a sufficient condition for the canonical ring of $X$ to be generated in degree less than or equal to $m$ but it is not a necessary condition. This is in contrast with the situation in dimension 2, where the converse of the result of Green is true, as it was proved by the authors in $[\mathrm{GP}]$.

\section{REFERENCES}

[Ca1] F. Catanese, Equations of pluriregular varieties of general type, Geometry today (Rome, 1984), 47-67, Progr. Math., 60, Birkhauser Boston, 1985. 
[Ca2] F. Catanese, Commutative algebra methods and equations of regular surfaces, Algebraic geometry, (Bucharest, 1982), 68-111, Lecture Notes in Math., 1056, Springer Berlin, 1984.

[Ci] C. Ciliberto, Sul grado dei generatori dell'anello di una superficie di tipo generale, Rend. Sem. Mat. Univ. Politec. Torino 41 (1983)

[GP] F.J. Gallego and B.P. Purnaprajna, On the canonical ring of covers of surfaces of minimal degree, preprint AG/0111052.

[G] M.L. Green, The canonical ring of a variety of general type, Duke Math. J. 49 (1982), 1087-1113.

[HM] D. Hahn and R. Miranda, Quadruple covers of algebraic varieties, J. Algebraic Geom. 8 (1999), 1-30.

[M] R. Miranda, Triple covers in Algebraic Geometry, Amer. J. Math. 107 (1985), 1123-1158. 